\title{
ASSOCIATIVITY PROPERTIES OF THE SYMPLECTIC SUM
}

\author{
Dusa McDuff and Margaret Symington
}

\begin{abstract}
In this note we apply a 4-fold sum operation to develop an associativity rule for the pairwise symplectic sum. This allows us to show that certain diffeomorphic symplectic 4-manifolds made out of elliptic surfaces are in fact symplectically deformation equivalent. We also show that blow-up points can be traded from one side of a symplectic sum to another without changing the symplectic deformation class of the resulting manifold.
\end{abstract}

\section{Introduction}

Recently there have been several new constructions for compact symplectic 4-manifolds $(X, \omega)$ as well as great progress (via Taubes-SeibergWitten theory) in understanding invariants for such manifolds. One of the main consequences of Taubes' work [10] is that the Gromov invariants of $(X, \omega)$ are invariants of the diffeomorphism type of $X$ rather than of its symplectomorphism type. It would be very interesting to understand whether or not a given diffeomorphism type can support two different symplectic structures. In fact, as yet no 4-dimensional example is known of a compact manifold with two distinct structures, though such examples were found by Ruan [7] in dimensions 6 and higher. The results presented here were developed to show that some possible candidates for such forms $\omega, \omega^{\prime}$ are in fact equivalent.

The appropriate notion of symplectic equivalence in the present context is that of weak deformation equivalence. Specifically, two symplectic forms $\omega, \omega^{\prime}$ on $X$ are deformation equivalent if there is a family of (possibly non-cohomologous) symplectic forms $\omega_{t}, 0 \leq t \leq 1$, such that $\omega_{0}=\omega$ and $\omega_{1}=\omega^{\prime}$, and two symplectic manifolds $(X, \omega),\left(X^{\prime}, \omega^{\prime}\right)$ are weakly deformation equivalent if there is a diffeomorphism $\phi: X \rightarrow X^{\prime}$ such that $\phi^{*}\left(\omega^{\prime}\right)$ is deformation equivalent to $\omega$. For example, a Kähler manifold supports a well-defined deformation class of symplectic forms since the set of Kähler forms compatible with a fixed complex structure is convex and hence path-connected.

Received May 1, 1996.

Partially supported by NSF grant DMS-9401443. 
Throughout this paper we restrict to the 4-dimensional case, though many of our results have higher dimensional analogues. When $S \subset X$ and $S^{\prime} \subset X^{\prime}$ are symplectically embedded surfaces in the 4-manifolds $X, X^{\prime}$, we write

$$
(X, S)=\left(X^{\prime}, S^{\prime}\right)
$$

if there is a symplectomorphism from $X$ to $X^{\prime}$ that takes $S$ to $S^{\prime}$, and

$$
(X, S) \cong\left(X^{\prime}, S^{\prime}\right)
$$

if the manifold/submanifold pairs are weakly deformation equivalent. (This means that the forms $\phi^{*}\left(\omega^{\prime}\right)$ and $\omega$ are equivalent under a symplectic deformation $\omega_{t}$ consisting of symplectic forms which are nondegenerate on $S$. For example, this is always the case if $\left(X, \omega_{t}\right)$ is Kähler and $S$ is a complex curve.) Finally, by a triple $(X, S, T)$ we mean a symplectic 4manifold $X$ with two symplectically embedded Riemann surfaces $S$ and $T$ which intersect transverally with positive orientation in a single point.

In [1] Gompf developed a pairwise symplectic sum, observing that when a pair of manifolds $X, X^{\prime}$ are summed along a pair of codimension two submanifolds, a transverse pair of submanifolds can be summed at the same time provided that certain conditions are satisfied. In 4-dimensions, the only pertinent condition is that the transverse surfaces must have positive intersection with the submanifolds along which the sum is being taken. Indeed, consider triples

$$
\left(X_{1}, S_{1}, T_{1}\right), \quad\left(X_{2}, S_{2}, T_{2}\right)
$$

for which

$$
g_{T_{1}}=g_{S_{2}}, \quad \iota_{T_{1}}=-\iota_{S_{2}}, \quad \int_{T_{1}} \omega_{1}=\int_{S_{2}} \omega_{2},
$$

where $g_{S}$ denotes the genus of $S$ and $\iota_{S}$ is the Chern number of its normal bundle, which in this setting is equal to the self-intersection number of $S$. Then one can form the pairwise sum of the manifold/submanifold pairs $\left(X_{1}, S_{1}\right)$ and $\left(X_{2}, T_{2}\right)$ along the symplectomorphic surfaces $T_{1}, S_{2}$ :

$$
\left(X_{1}, S_{1}\right) \#_{T_{1}=S_{2}}\left(X_{2}, T_{2}\right)=\left(X_{1} \#_{T_{1}=S_{2}} X_{2}, S_{1} \# T_{2}\right)
$$

where $S_{1} \# T_{2}$ is the connected sum of surfaces isotopic to $S_{1}, T_{2}$. This sum is described in detail in $\S 2$. (Our notation in which $T_{1}$ is glued to $S_{2}$ might seem a little awkward, but will prove to be very convenient.)

Our first observation is that the 4-fold sum operation, which is developed by the second author in [9], is invariant under cyclic permutations. The 4-fold sum is possible when four symplectic triples

$$
\left(X_{1}, S_{1}, T_{1}\right), \quad\left(X_{2}, S_{2}, T_{2}\right), \quad\left(X_{3}, S_{3}, T_{3}\right), \quad\left(X_{4}, S_{4}, T_{4}\right)
$$


are such that each $\left(X_{i}, S_{i}, T_{i}\right)$ can be summed to $\left(X_{i+1}, S_{i+1}, T_{i+1}\right)$ along $T_{i}, S_{i+1}$ as above, where $i$ is understood $\bmod 4$. To form the sum, remove all eight surfaces $S_{i}, T_{i}$ and naively start making symplectic pairwise sums:

$$
\begin{array}{cl}
X_{1} \#_{T_{1}=S_{2}} X_{2}, & X_{2} \#_{T_{2}=S_{3}} X_{3} \\
X_{3} \#_{T_{3}=S_{4}} X_{4} & X_{4} \#_{T_{4}=S_{1}} X_{1} .
\end{array}
$$

We explain in $\S 2$ how these sums continue into the neighborhoods of the intersection points to yield a smooth symplectic manifold, the 4-fold sum. Viewing each pair $S_{i}, T_{i}$ as one immersed manifold, the 4 -fold sum is a very simple example of an extension of the symplectic sum to a sum along immersed manifolds, a possibility suggested by Gromov [3]. In $\S 3$ we prove:

Proposition 1.1 (4-fold sum rule). If triples $\left(X_{i}, S_{i}, T_{i}\right), 1 \leq i \leq 4$ are such that for all $i(\bmod 4)$

$$
g_{T_{i}}=g_{S_{i+1}}, \quad \iota_{T_{i}}=-\iota_{S_{i+1}}, \quad \int_{T_{i}} \omega_{i}=\int_{S_{i+1}} \omega_{i+1}
$$

then

$$
\begin{aligned}
\left(X_{1} \#_{T_{1}=S_{2}} X_{2}\right) \#_{S_{1} \# T_{2}=S_{3} \# T_{4}}\left(X_{3} \#_{T_{3}=S_{4}} X_{4}\right) \\
=\left(X_{4} \#_{T_{4}=S_{1}} X_{1}\right) \#_{S_{4} \# T_{1}=S_{2} \# T_{3}}\left(X_{2} \#_{T_{2}=S_{3}} X_{3}\right)
\end{aligned}
$$

Using this we prove an associativity rule for a sum of three triples $\left(X_{i}, S_{i}, T_{i}\right), i=1,2,3$. Before stating this, we note that if $S$ and $T$ are symplectic submanifolds which intersect positively along a symplectic submanifold it is always possible to construct a symplectic submanifold in the class $[S]+[T]$ which equals $S \cup T$ except near the intersection $S \cap T$. We will think of this manifold as the desingularization of $S \cup T$ and will denote it by $S+T$. It is not hard to show that $S+T$ is well-defined up to symplectic isotopy.

Proposition 1.2 (Associativity). Suppose that for $i=1,2,3(\bmod 3)$

$$
\begin{array}{ll}
g_{T_{i}}=g_{S_{i+1}}, & \int_{T_{i}} \omega_{i}=\int_{S_{i+1}} \omega_{i+1}, \quad \text { and } \\
\iota_{T_{1}}=-\iota_{S_{2}}, & \iota_{T_{2}}=-\iota_{S_{3}}, \quad \iota_{T_{3}}=-\left(\iota_{S_{1}}+2\right) .
\end{array}
$$

Then

$\left(X_{1} \#_{T_{1}=S_{2}} X_{2}\right) \#_{S_{1} \# T_{2}=S_{3}+T_{3}} X_{3}=X_{1} \#_{S_{1}+T_{1}=S_{2} \# T_{3}}\left(X_{2} \#_{T_{2}=S_{3}} X_{3}\right)$. 
Proof. (Sketch) To prove the result up to deformation we will find a triple $\left(X_{4}, S_{4}, T_{4}\right)$ which, when summed with either $X_{3}$ or $X_{1}$, yields a manifold symplectically deformation equivalent to the original summand and containing $S_{3} \# T_{4}$ or $S_{4} \# T_{1}$ as a representative of the class $\left[S_{3}\right]+\left[T_{3}\right]$ or $\left[S_{1}\right]+\left[T_{1}\right]$ respectively.

The triple we will use is $\left(W_{g}, \Gamma_{-k+2}, \Gamma_{k}\right)$ where $W_{g}$ is a ruled surface over a Riemann surface of genus $g$ with a pair of sections $\Gamma_{-k+2}, \Gamma_{k}$ that intersect positively in one point. Indeed, we prove in $\S 3$ :

Lemma 1.3. Given any triple $(X, S, T)$, if $(g, k)=\left(g_{S},-\iota_{S}\right)$ then the symplectic sum with the triple $\left(W_{g}, \Gamma_{-k+2}, \Gamma_{k}\right)$ yields

$$
(X, S+T) \cong\left(W_{g} \#_{\Gamma_{k}=S} X, \Gamma_{-k+2} \# T\right) .
$$

Analogously, if $(g, k)=\left(g_{T}, \iota_{T}+2\right)$ then

$$
(X, S+T) \cong\left(X \#_{T=\Gamma_{-k+2}} W_{g}, S \# \Gamma_{k}\right) .
$$

Granted this, we apply the 4 -fold sum rule with $\left(X_{4}, S_{4}, T_{4}\right)=$ $\left(W_{g}, \Gamma_{-k+2}, \Gamma_{k}\right)$ where

$$
g=g_{S_{1}}=g_{T_{3}}, \quad k=-\iota_{S_{1}}=\iota_{T_{3}}+2 .
$$

It is easy to check that the 4 -fold sum is well-defined. Moreover, by Lemma 1.3

$$
\begin{aligned}
& \left(X_{4} \#{ }_{T_{4}=S_{1}} X_{1}, S_{4} \# T_{1}\right) \cong\left(X_{1}, S_{1}+T_{1}\right) \text { and } \\
& \left(X_{3} \#_{T_{3}=S_{4}} X_{4}, S_{3} \# T_{4}\right) \cong\left(X_{3}, S_{3}+T_{3}\right) .
\end{aligned}
$$

This proves the two manifolds are deformation equivalent. The proof that they are symplectomorphic is given in $\S 4$.

Remark 1.4. In order to prove Proposition 1.2 up to symplectomorphism rather than deformation equivalence we will need a thickening/thinning procedure which is described in $\S 4$. By thickening and thinning we can build the necessary $X_{4}=W_{g}$ out of pieces removed from the other $X_{i}$ 's.

One application of these results is to prove the symplectic equivalence of two manifolds which are constructed out of elliptic surfaces. Let $E(n)$ be the elliptic surface which is the $n$-fold branched cover of $E(1)=\mathbb{C} P^{2} \# 9 \overline{\mathbb{C P}}^{2}$ along a pair of fibers. Then $E(n)$ contains 9 sections $\Sigma_{-n}$ which are $n$-fold covers of the exceptional spheres in $E(1)$ and so have self-intersection $-n$. In terms of the symplectic sum, we can inductively define

$$
\left(E(n), \Sigma_{-n}\right) \cong\left(E(n-1) \#_{F_{n-1}=F_{1}} E(1), \Sigma_{-n+1} \# \Sigma_{-1}\right)
$$


where $F_{k}$ is a generic fiber in $E(k)$. When $n=4$ one can sum along the section $\Sigma_{-4}$ and a quadric curve $Q$ in $\mathbb{C} P^{2}$ to form the manifold $E(4) \#_{\Sigma_{-4}=Q} \mathbb{C} P^{2}$ which is not diffeomorphic to any complex surface (see Gompf [1]). On the other hand, when $n=3$ there is a torus $T_{-1}$ in the homology class $\left[\Sigma_{-3}\right]+\left[F_{3}\right]$. Since

$$
T_{-1} \cdot T_{-1}=\Sigma_{-3} \cdot \Sigma_{-3}+2 \Sigma_{-3} \cdot F_{3}=-1,
$$

$E(3)$ can be summed with $Y=\mathbb{C} P^{2} \# 8 \overline{\mathbb{C} P}^{2}$ along the tori $T_{-1}, T_{1}$ where $T_{1}$ is a torus of self-intersection +1 in $Y$ which is obtained from an elliptic curve in $\mathbb{C} P^{2}$ through the 8 blown up points.

Stipsicz [8] proved that the Donaldson invariants of these two manifolds

$$
E(4) \#_{\Sigma_{-4}=Q} \mathbb{C} P^{2}, \quad E(3) \#_{T_{-1}=T_{1}} Y
$$

are the same and Gompf [2] has shown using Kirby calculus that they are diffeomorphic. We show here that the manifolds are symplectically deformation equivalent. (Observe that, since they are built out of Kähler surfaces, both manifolds have symplectic forms well-defined up to deformation.)

Proposition 1.5. The manifolds $E(4) \#_{\Sigma_{-4}=Q} \mathbb{C} P^{2}$ and $E(3) \#_{T_{-1}=T_{1}} Y$ are symplectically deformation equivalent.

Proof. First, scale the symplectic forms on $E(1), E(3)$ so that the symplectic areas of the fibers are equal. Next, adjust these forms (by pulling back suitable forms from the base of the elliptic fibrations) to make the sections $\Sigma_{-1}, \Sigma_{-3}$ have the same symplectic area $a_{\Sigma}$, and choose a symplectic form on $\mathbb{C} P^{2}$ such that the symplectic area of a line equals $a_{\Sigma}$. Then, if we take

$$
\begin{aligned}
& \left(X_{1}, S_{1}, T_{1}\right)=\left(E(3), \Sigma_{-3}, F_{3}\right) \\
& \left(X_{2}, S_{2}, T_{2}\right)=\left(E(1), F_{1}, \Sigma_{-1}\right) \\
& \left(X_{3}, S_{3}, T_{3}\right)=\left(\mathbb{C} P^{2}, L_{1}, L_{2}\right)
\end{aligned}
$$

where $L_{1}, L_{2}$ are two lines in $\mathbb{C} P^{2}$, the 3 -fold sums of Proposition 1.2 are defined. Further,

$$
\begin{aligned}
& \left(X_{1} \#_{T_{1}=S_{2}} X_{2}\right) \#_{S_{1} \# T_{2}=S_{3}+T_{3}} X_{3} \\
& =\left(E(3) \#_{F_{3}=F_{1}} E(1)\right) \#_{\Sigma_{-4}=Q} \mathbb{C} P^{2}=E(4) \#_{\Sigma_{-4}=Q} \mathbb{C} P^{2} .
\end{aligned}
$$

On the other hand

$$
\begin{aligned}
& X_{1} \#_{S_{1}+T_{1}=S_{2} \# T_{3}}\left(X_{2} \#_{T_{2}=S_{3}} X_{3}\right) \\
& =E(3) \#_{T_{-1}=F_{1} \# L_{2}}\left(E(1) \#_{\Sigma_{-1}=L_{1}} \mathbb{C} P^{2}\right)=E(3) \#_{T_{-1}=T_{1}} Y,
\end{aligned}
$$


where the last equivalence holds because the sum with $\mathbb{C} P^{2}$ is just a symplectic blow-down of $\Sigma_{-1}$ and so takes $E(1)$ to $Y$ and $F_{1}$ to $T_{1}$.

Another application in the same spirit uses properties of the ruled surfaces $W_{g}$ to show that blow-up points can be traded from one side of a symplectic sum to the other without changing the deformation class of the symplectic structure. McCarthy and Wolfson [6] noted that a standard handle trading argument shows that this can be done up to diffeomorphism, as explained in detail by Gompf in Lemma 5.1 of [1]. However, these authors left open the question of symplectic equivalence. We prove

Proposition 1.6. Consider symplectic pairs $(X, S),\left(X^{\prime}, S^{\prime}\right)$ such that

$$
\iota_{S}=-\iota_{S^{\prime}}+1, \quad g_{S}=g_{S^{\prime}} .
$$

Let $\widetilde{X}$ be the blow-up of $X$ at a point of $S$ and $\widetilde{S}$ the proper transform of $S$, and similarly for $\left(\widetilde{X}^{\prime}, \widetilde{S}^{\prime}\right)$. Then

$$
X \#_{S=\widetilde{S}^{\prime}} \widetilde{X}^{\prime} \cong \widetilde{X} \#_{\widetilde{S}=S^{\prime}} X^{\prime} .
$$

Remark 1.7. (i) Because the blow-down operation on $\widetilde{X}$ may be interpreted as a sum with $\mathbb{C} P^{2}$, an equivalent way of stating this (which shows its similarity to the associativity rule) is:

$$
\left(\widetilde{X} \#_{E=L_{1}} \mathbb{C} P^{2}\right) \#_{\widetilde{S} \# L_{2}=\widetilde{S}^{\prime}} \widetilde{X}^{\prime} \cong \widetilde{X} \#_{\widetilde{S}=L_{2} \# \widetilde{S}^{\prime}}\left(\mathbb{C} P^{2} \#_{L_{1}=E^{\prime}} \widetilde{X}^{\prime}\right) .
$$

Here $E, E^{\prime}$ denote the exceptional spheres in $\widetilde{X}, \widetilde{X}^{\prime}$, and $L_{1}, L_{2}$ are lines in $\mathbb{C} P^{2}$.

(ii) The invariance of the symplectic structure under the trading of blow-up points can be at most up to deformation equivalence since it is impossible to fix the symplectic areas of $S$ and $S^{\prime}$ in such a way that both sums can be performed. To see this, observe that the area of the proper transform of a surface (after a symplectic blow-up) is less than that of the original surface, so the symplectic sum along $S, \widetilde{S}^{\prime}$ requires $\int_{S} \omega<\int_{S^{\prime}} \omega^{\prime}$ while a sum along $\widetilde{S}, S^{\prime}$ requires the reverse inequality.

As another application, we show that rational blow-down of a -4-sphere gives nothing new if this sphere is the blow-up of a -3 -sphere. Again, this was proved by Gompf as far as concerns diffeomorphism type.

Corollary 1.8. Let $S \subset X$ be a sphere with $\iota_{S}=-3$ and let $Q$ be a quadric curve in $\mathbb{C} P^{2}$. Then

$$
\widetilde{X} \# \widetilde{S}=Q_{\mathbb{C}} P^{2} \cong X
$$


Proof. Proposition 1.6 shows that $\widetilde{X} \#_{\widetilde{S}=Q} \mathbb{C} P^{2} \cong X \#_{S=\widetilde{Q}} \widetilde{\mathbb{C} P^{2}}$. But, we may think of $\widetilde{\mathbb{C} P^{2}}$ as the projectivization $\mathbb{P}\left(\mathbb{C} \oplus \mathcal{L}_{3}\right)$ of a complex rank 2 bundle over $\mathbb{C} P^{1}$ where $\mathcal{L}_{3}$ is a complex line bundle of Chern number 3. Thus $\widetilde{\mathbb{C} P^{2}}$ is the union of a neighborhood of the section $\widetilde{Q}$ with a neighborhood of a -3 section, and it follows immediately that $X \#_{S=\widetilde{Q}} \widetilde{\mathbb{C} P} P^{2} \cong X$.

\section{The 4-fold sum}

The associativity rule (Proposition 1.2) is a consequence of the fact that a simple version of the 4 -fold sum is equivalent to a sequence of three symplectic sums (two of which are pairwise) performed in either of two ways. We begin with a description of the pairwise symplectic sum in terms of images under the moment map for a local torus action.

2.1. The symplectic and pairwise sums. Given a pair of symplectic submanifolds $S_{i} \subset\left(X_{i}, \omega_{i}\right), i=1,2$ and a symplectomorphism $\phi: S_{1} \rightarrow$ $S_{2}$, one can perform a symplectic sum of $X_{1}$ and $X_{2}$ along $S_{1}$ and $S_{2}$ provided the normal numbers (Chern numbers of the normal bundles) of the submanifolds sum to zero. This operation was originally introduced by Gromov [3] and can be viewed as an inverse to Lerman's symplectic cutting procedure [5]. Observe first that a codimension two symplectic submanifold $S$ in $X$ always has a tubular neighborhood $\mathcal{N}_{S}$ that admits a Hamiltonian circle action with fixed point set $S$. Moreover, one can clearly extend the induced action on $\mathcal{N}_{S}-S$ to a free Hamiltonian action on a collar neighborhood $\overline{\mathcal{N}}_{S}$ of the boundary in an appropriate closure $\overline{X-S}$ of $X-S$. Here $\mathcal{N}_{S}$ is an open disc bundle over $S$, and $\overline{\mathcal{N}}_{S}$ is the associated bundle with fibers $[0,1) \times S^{1}$ so that its boundary $\partial_{S}=\{0\} \times S^{1}$ is a circle bundle over $S$. Furthermore, this boundary $\partial_{S}$ is a level set of the Hamiltonian which generates the free action, and its symplectic reduction is $S$ itself. Now, the way to form the sum

$$
X_{1} \#_{S_{1}=S_{2}} X_{2}
$$

is to remove the submanifolds $S_{i}$, take the closures $\overline{X_{i}-S_{i}}$ as described above, and then identify the boundaries via an orientation reversing diffeomorphism $\bar{\phi}: \partial_{S_{1}} \rightarrow \partial_{S_{2}}$ that covers $\phi$ and thus matches the characteristic foliations (along which the symplectic forms are degenerate).

Remark 2.1. Note that the diffeomorphism class of the summed manifold depends on the choice of the fiber isotopy class of the map $\bar{\phi}$. In the examples we consider in this paper we sum along fibers in elliptic surfaces and use the canonical framings to get the boundary identifications. 


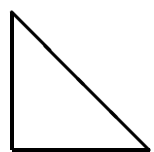

(a)

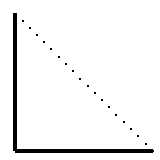

(b)

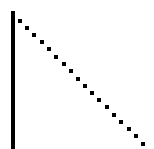

(c)

FigURE 1. Images of moment maps.

In order to describe the pairwise sum in a similar way, one needs to use torus actions rather than circle actions. Recall that when a closed 4-manifold admits a Hamiltonian action of $T^{2}$, the image of an associated moment map is a convex polytope in $\mathbb{R}^{2}$. The preimages of points on the interior of the polytope are tori, while the preimages of points on an edge or vertices are circles or points respectively. We use the convention that replacing a solid line segment in the image of a moment map by a heavy dotted line segment corresponds to replacing the submanifold $S$ (the preimage of the solid line) with the associated boundary $\partial_{S}$. A light dotted line indicates an open boundary. For instance,

Example 2.2. Figure 1 shows the image under the moment map for (a) $\mathbb{C} P^{2}$, (b) the open ball obtained by removing a $\mathbb{C} P^{1}=S$ and (c) its closure, a closed ball with boundary $\partial_{S}$.

For the purposes of this paper we want to keep track of the effect of the symplectic sum on transverse symplectic submanifolds. If two symplectic surfaces $S, T$ intersect transversely and positively, then one of them can be perturbed, via an isotopy through symplectic surfaces, so that the intersection is orthogonal with respect to the symplectic structure (see [1] Lemma 2.3, for example).

Consider a triple $(X, S, T)$ as in $\S 1$. Then in a neighborhood $\mathcal{N}_{x}$ of the intersection point $\{x\}=S \cap T$ there is a Hamiltonian $T^{2}$ action such that the first $S^{1}$ factor has fixed point set $S \cap \mathcal{N}_{x}$ and the second has fixed point set $T \cap \mathcal{N}_{x}$. Thus the image of the moment map is a neighborhood of a corner in a square. We may close $X-(S \cup T)$ by adding a boundary (with corner) to get a compact symplectic manifold on which there is a free local $T^{2}$ action acting in a neighborhood of the corner.

For triples $\left(X_{i}, S_{i}, T_{i}\right), i=1 \cdots 4$ we choose neighborhoods of the intersection points whose images under the moment map are as in Figure 2(a), where the slopes of the slanted edges are $-\iota_{S_{i}}$ and $-1 / \iota_{T_{i}}$.

Remark 2.3. These images show the correct convexity (or concavity) of the symplectic neighborhoods. Indeed, when the normal number of a sub- 
manifold is positive, removing a tubular neighborhood whose boundary is a level set of the Hamiltonian and taking the symplectic reduction of the newly formed boundary yields a surface whose area is smaller than that of the original surface.

In order for our notation to be consistent with that used for the 4fold sum, the summing operation identifies $T_{i}$ with $S_{i+1}(\bmod 4)$. We assume that $S_{i}$ intersects $T_{i}$ orthogonally in $x_{i}$ and that the gluing map $\phi_{i}: T_{i} \rightarrow S_{i+1}$ takes $x_{i}$ to $x_{i+1}$. Then the first diagram of Figure 2(b) shows the image under the moment map of these neighborhoods after the symplectic sum has been taken along $T_{1}, S_{2}$ using the symplectomorphism $\phi_{1}$. The bold horizontal line at the bottom consists of points with preimage equal to a circle and so is a neighborhood in the connected sum $S_{1} \# T_{2}$ of the attaching circle. The heavy dotted vertical line segment represents the normal 2-disc bundle over the attaching circle, or equivalently, the intersection of the neighborhood $\mathcal{N}_{x}$ and the identified boundaries associated to the submanifolds $T_{1}, S_{2}$. The second diagram is a similar picture of the sum along $T_{3}, S_{4}$. Since any positive intersection can be made orthogonal via an isotopy of one of the intersecting surfaces, Figure 2(b) makes the following lemma clear. It is a rephrasing of the 4-dimensional case of Theorem 1.4 in Gompf [1].

Lemma 2.4. Consider two triples $\left(X_{i}, S_{i}, T_{i}\right), i=1,2$. If $T_{1}$ and $S_{2}$ have the same area and genus, and $\iota_{T_{1}}=-\iota_{S_{2}}$, then in the manifold $X_{1} \#_{T_{1}=S_{2}} X_{2}$ there is a symplectic surface that is the connected sum of surfaces isotopic to $S_{1}$ and $T_{2}$. The normal number of the surface $S_{1} \# T_{2}$ is the sum of the normal numbers of $S_{1}$ and $T_{2}$.

2.2. The 4-fold sum. The 4 -fold sum is a sum along the four pairs of surfaces in four triples $\left(X_{i}, S_{i}, T_{i}\right), i=1, \cdots 4$ such that for each $i$,

- $S_{i} \cap T_{i}=\left\{x_{i}\right\}$ and the intersection is orthogonal with respect to $\omega_{i}$,

- $\int_{T_{i}} \omega_{i}=\int_{S_{i+1}} \omega_{i+1}$ and

- $\iota_{T_{i}}=-\iota_{S_{i+1}}$

where the subscripts are taken mod 4. Call such a collection of triples admissible.

Because the normal numbers of each pair of symplectomorphic surfaces sum to zero, it is possible to sum along all four pairs $T_{i}, S_{i+1}$. Trying to do these sums simultaneously leads to the following definition:

Definition 2.5. Given an admissible collection of triples $\left(X_{i}, S_{i}, T_{i}\right), i=$ $1, \cdots 4$ and symplectomorphisms $\phi_{i}: T_{i} \rightarrow S_{i+1}$ such that $\phi_{i}\left(x_{i}\right)=x_{i+1}$ 
(a)

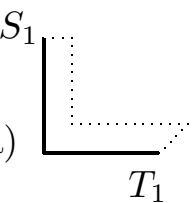

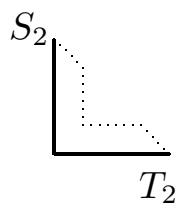
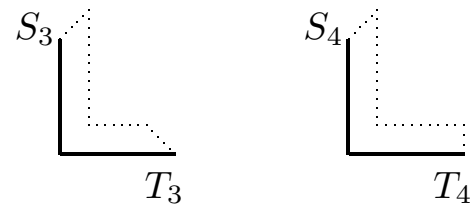

(b)
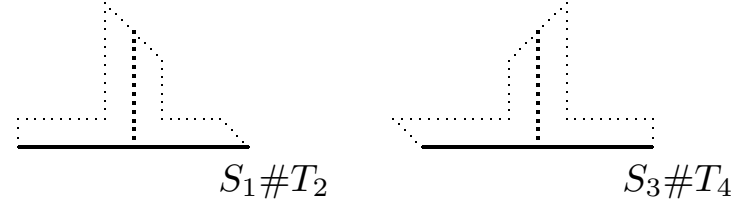

(c)

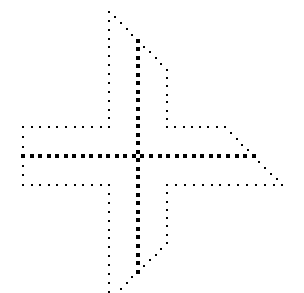

Figure 2. The symplectic sum.

$(\bmod 4)$, let

$$
\overline{X_{i}-\left(S_{i} \cup T_{i}\right)}
$$

be the closure of $X_{i}-\left(S_{i} \cup T_{i}\right)$ with free local $T^{2}$ action as described above. Then, choosing orientation reversing diffeomorphisms $\bar{\phi}_{i}$ that cover the $\phi_{i}$, we define

$$
\sqcup_{i=1}^{4} \overline{X_{i}-\left(S_{i} \cup T_{i}\right)} / \bar{\phi}_{i}
$$

to be the 4-fold sum of the $X_{i}$ along the surfaces $T_{i}, S_{i+1}$.

To see that this construction yields a smooth symplectic manifold, notice that it is equivalent to a sequence of three symplectic sums. Indeed, given a set of four triples as in the definition, by Lemma 2.4 we can use the maps $\bar{\phi}_{1}$ and $\bar{\phi}_{3}$ to form pairwise sums along the pairs $T_{1}, S_{2}$ and $T_{3}, S_{4}$ to yield two manifolds which contain surfaces $S_{1} \# T_{2}$ and $S_{3} \# T_{4}$ respectively. These surfaces have the same area and genus, and have normal numbers $\iota_{S_{1}}+\iota_{T_{2}}$ and $\iota_{S_{3}}+\iota_{T_{4}}=-\iota_{T_{2}}-\iota_{S_{1}}$ respectively. Therefore we can form the symplectic sum along this new pair. In fact, to perform this sum, we can use the diffeomorphisms $\bar{\phi}_{2}$ and $\bar{\phi}_{4}$ which agree on the overlapping circle. Figure 2 shows these sums, keeping track of the convexity of all of the tubular neighborhoods. Thus, the only place the 4-fold sum might not have been a smooth symplectic manifold it is in fact symplectomorphic to the product of $T^{2}$ and a domain in $\mathbb{R}^{2}$ with the symplectic structure of 
$T^{*} T^{2}$. A precise statement and proof of this fact is given in Symington [9].

Proposition 1.1 follows immediately since the 4-fold sum is certainly also equivalent to first summing pairwise along the surfaces $T_{2}, S_{3}$ and $T_{4}, S_{1}$ and then afterwards summing the resulting manifolds along the surfaces $S_{2} \# T_{3}, S_{4} \# T_{1}$.

\section{Proofs of the main results}

We begin by describing the manifolds $W_{g}$ of Lemma 1.3 . For each genus $g$ and integer $n \geq 1$ let $W_{g}=W_{g, n}$ be an $S^{2}$ bundle over a Riemann surface of genus $g$. Make $W_{g}$ be the trivial bundle (i.e. a product) if $n$ is even, and the nontrivial bundle when $n$ is odd. In either case, there is a unique symplectic structure on the ruled surface $W_{g}$ up to deformation. (In fact, by [4] symplectic forms in a given cohomology class on $W_{g}$ are unique up to isotopy.) We choose a symplectic structure on $W_{g}$ so that the manifold contains symplectic sections $\Gamma_{-n+2 p}$ of self-intersection $-n+2 p$ for all $p \geq 0$. Then in particular there are symplectic sections $\Gamma_{-k+2}$ and $\Gamma_{k}$ which intersect once transversally (and positively) for each $-n \leq k \leq n+2$.

To be explicit, realize $W_{g}$ as the projectivization of the complex rank 2 bundle $\mathbb{C} \oplus \mathcal{L}_{n}$ as in Corollary 1.8. Then $\Gamma_{n}$ and $\Gamma_{-n}$ are the holomorphic (and therefore symplectic) sections at zero and infinity. Observe that they are disjoint. If $F$ is a fiber then $\left[\Gamma_{-n}\right]$ and $[F]$ form a basis for the homology of $W_{g}$ such that $\left[\Gamma_{n}\right]=\left[\Gamma_{-n}\right]+n[F]$. Because the fiber can also be realized as a holomorphic curve it is clear that there are symplectic sections $\Gamma_{-n+2} \in\left[\Gamma_{-n}\right]+[F]$ and $\Gamma_{n-2} \in\left[\Gamma_{-n}\right]+(n-1)[F]$. Notice that the ruled surface $W_{g}$ is also the total space of a Hamiltonian $S^{1}$-action with fixed point sets $\Gamma_{n}$ and $\Gamma_{-n}$.

Henceforth, we assume that $W_{g}=W_{g, n}$ with $n$ sufficiently large that any sections referred to can be (and are) taken to be symplectic. Note that the parity of the self-intersection numbers of the sections will in all cases make it clear whether or not $W_{g}$ is the trivial bundle.

We claim that the manifold $W_{g}$ has the exact properties we need in order to prove Lemma 1.3. First of all, because the sections of opposite self-intersection are disjoint, $\left[\Gamma_{k}\right] \cdot\left(\left[\Gamma_{-k}\right]+[F]\right)=1$, so the sections $\Gamma_{k}$ and $\Gamma_{-k+2}$ intersect once positively. Second of all, we can view $W_{g}$ either as the union of symplectic neighborhoods of $\Gamma_{k}$ and $\Gamma_{-k}$ or of $\Gamma_{k-2}$ and $\Gamma_{-k+2}$, thanks to the fibration by symplectic spheres.

We now show that summing $\left(W_{g}, \Gamma_{-k+2}, \Gamma_{k}\right)$ with a triple $(X, S, T)$ desingularizes the intersecting submanifolds $S, T$.

Proof of Lemma 1.3. We will show that if $S \subset X$ has genus $g$ and $\iota_{S}=-k$, 
then

$$
(X, S+T) \cong\left(W_{g} \#{ }_{\Gamma_{k}=S} X, \Gamma_{-k+2} \# T\right) .
$$

The other identity then follows by replacing $k$ by $-k+2$ and interchanging the roles of $S, T$.

Now, it is obvious that $X \cong W_{g} \#_{\Gamma_{k}=S} X$, i.e. $W_{g}$ is a neutral element for the symplectic sum operation in the category of symplectic deformation equivalence classes. As for the statement about the submanifold $S+T$, observe that we have

$$
(X, S, T) \cong\left(W_{g} \#{ }_{\Gamma_{k}=S} X, \Gamma_{-k}, F \# T\right)
$$

Hence

$$
\begin{aligned}
(X, S+T) & \cong\left(W_{g} \#_{\Gamma_{k}=S} X, \Gamma_{-k}+F \# T\right) \\
& \cong\left(W_{g} \#_{\Gamma_{k}=S} X,\left(\Gamma_{-k}+F\right) \# T\right) \\
& \cong\left(W_{g} \#_{\Gamma_{k}=S} X, \Gamma_{-k+2} \# T\right)
\end{aligned}
$$

where the second equivalence holds because $\Gamma_{-k}$ is disjoint from $\Gamma_{k}$.

To show that one can trade blow-up points we use a pair of disjoint sections in the ruled surface $W_{g}$, rather than a pair that intersect once.

Proof of Proposition 1.6. Let $W=W_{g}$ be a ruled surface with disjoint sections $\Gamma_{ \pm(k+1)}$ and let $W^{\prime}=W_{g}^{\prime}$ be one with disjoint sections $\Gamma_{ \pm k}$. Thus, one of these manifolds $W, W^{\prime}$ will be a trivial fibration and the other one non-trivial. Blow $W$ up at a point of $\Gamma_{k+1}$ and blow $W^{\prime}$ up at a point of $\Gamma_{-k}^{\prime}$. It is well known that there is a diffeomorphism that realizes $\widetilde{W} \cong \widetilde{W}^{\prime}$, taking $\widetilde{\Gamma}_{k+1}$ to $\Gamma_{k}^{\prime}$, and $\Gamma_{-k-1}$ to $\widetilde{\Gamma}_{-k}^{\prime}$. Recall that the symplectic structures on these manifolds are equivalent up to deformation. Thus

$$
\left(\widetilde{W}, \widetilde{\Gamma}_{k+1}, \Gamma_{-k-1}\right) \cong\left(\widetilde{W}^{\prime}, \Gamma_{k}^{\prime}, \widetilde{\Gamma}_{-k}^{\prime}\right)
$$

Letting $k=-\iota_{S}-1$, we can deform $(X, S) \cong\left(X \#_{S=\Gamma_{-k-1}} W, \Gamma_{k+1}\right)$ and we clearly have:

$$
\begin{aligned}
(\widetilde{X}, \widetilde{S}) & \cong\left(X \#_{S=\Gamma_{-k-1}} \widetilde{W}, \widetilde{\Gamma}_{k+1}\right) \\
& \cong\left(X \#_{S=\widetilde{\Gamma}_{-k}^{\prime}} \widetilde{W^{\prime}}, \Gamma_{k}^{\prime}\right)
\end{aligned}
$$


Hence, because $\widetilde{\Gamma}_{-k}^{\prime}$ and $\Gamma_{k}^{\prime}$ are disjoint in $\widetilde{W}^{\prime}$,

$$
\begin{aligned}
\widetilde{X} \#_{\widetilde{S}=S^{\prime}} X^{\prime} & \cong\left(X \#_{S=\widetilde{\Gamma}_{-k}^{\prime}} \widetilde{W^{\prime}} \#_{\Gamma_{k}^{\prime}=S^{\prime}} X^{\prime}\right. \\
& \cong X \#_{S=\widetilde{\Gamma}_{-k}^{\prime}}\left(\widetilde{W}^{\prime} \#_{\Gamma_{k}^{\prime}=S^{\prime}} X^{\prime}\right) \\
& \cong X \#_{S=\widetilde{S}^{\prime}} \widetilde{X}^{\prime} .
\end{aligned}
$$

Note that the effect of deforming $(X, S)$ to $\left(X \#_{S=\Gamma_{-k-1}} W, \Gamma_{k+1}\right)$ is to localize the argument near $S$, i.e. we represent a neighborhood of $S$ in $X$ as a neighborhood of $\Gamma_{k+1}$ in $W$ and then work in $W$.

\section{Thickening and thinning}

In order to prove the associativity rule up to symplectomorphism we need a refinement of Lemma 1.3. The notation $W_{g, \varepsilon}$ will mean that the symplectic form on $W_{g}$ has been scaled so that the fiber has symplectic area $\varepsilon>0$. By [4] the symplectic form on $W_{g, \varepsilon}$ is then determined up to symplectomorphism (even isotopy) by specifying the area of one section. (The only condition on this area is the following: if $\omega\left(\Gamma_{k}\right)=a$ we need $a>k \varepsilon / 2$, unless $g=0, k$ is odd, in which case we need $a>(k+1) \varepsilon / 2$. Thus, given $a$, this is satisfied for sufficiently small $\varepsilon$.)

The next lemma says that $W_{2 \varepsilon}$ can be thought of as the sum of two copies of $W_{\varepsilon}$.

Lemma 4.1. If symplectic forms on two copies $W_{g, \varepsilon}^{i}, i=1,2$ of $W_{g, \varepsilon}$ are chosen so that the area of the section $\Gamma_{k}^{1}$ in the first equals the area of $\Gamma_{-k}^{2}$ in the second, then

$$
\left(W_{g, \varepsilon}^{1} \#_{\Gamma_{k}^{1}=\Gamma_{-k}^{2}} W_{g, \varepsilon}^{2}, \Gamma_{-k}^{1}, \Gamma_{k}^{2}\right)=\left(W_{g, 2 \varepsilon}, \Gamma_{-k}, \Gamma_{k}\right) .
$$

Proof. We use Lerman's symplectic cutting procedure [5] to show that $W_{g, 2 \varepsilon}$ decomposes as a sum of this kind. Choose a Hamiltonian function $f$ on $W_{g, 2 \varepsilon}$ with fixed point sets $\Gamma_{-k}=f^{-1}(0)$ and $\Gamma_{k}=f^{-1}(2 \varepsilon)$. Then both of the ruled manifolds obtained by cutting $W_{g, 2 \varepsilon}$ along the $S^{1}$-invariant hypersurface $f^{-1}(\varepsilon)$ and taking the $S^{1}$ reduction along the boundaries of $f^{-1}([0, \varepsilon]), f^{-1}([\varepsilon, 2 \varepsilon])$ have fiber of size $\varepsilon$ and so may be identified with the manifolds $W_{g, \varepsilon}^{1}, W_{g, \varepsilon}^{2}$.

Given a triple $(X, S, T)$ with symplectic structure $\omega$ we write

$$
\left(\mathcal{T}_{S}^{-}(X), S^{-}, T^{-}\right)
$$

for the (deformation equivalent) manifold formed by "thinning" $X$ along $S$ by the amount $\varepsilon$, for some sufficiently small $\varepsilon$ (which determines $\mathcal{T}_{S}^{-}(X)$ up to symplectomorphism). In terms of the language of $\S 2$, we remove 
an $S^{1}$-invariant open tubular neighborhood $\mathcal{N}_{S}$ of $S$ with fiber of area $\varepsilon$, and then reduce the boundary of $X-\mathcal{N}_{S}$ by the $S^{1}$ action. The surface $S^{-}$is the symplectic reduction of the boundary. Because $T$ coincides near $S$ with an orthogonal symplectic fiber of $\mathcal{N}_{S}$, the manifold $T^{-}$is just $T$ with a disk of area $\varepsilon$ removed and the circle boundary collapsed to a point. Indeed, we have

$$
(X, S, T)=\left(\mathcal{T}_{S}^{-}(X) \#_{S^{-}=\Gamma_{k}} W_{g, \varepsilon}, \Gamma_{-k}, T^{-} \# F\right)
$$

where $g=g_{S}, k=-\iota_{S}$ and $F$ is a fiber of the ruled surface $W_{g, \varepsilon}$. Letting $\omega^{-}$be the symplectic form on $\mathcal{T}_{S}^{-}(X)$, we have

$$
\int_{\mathcal{T}_{S}^{-}(X)}\left(\omega^{-}\right)^{2}<\int_{X} \omega^{2}, \quad \int_{S^{-}} \omega^{-}=-\varepsilon \iota_{S}+\int_{S} \omega, \quad \int_{T^{-}} \omega^{-}=-\varepsilon+\int_{T} \omega .
$$

Analogously, we can "thicken" along the surface $S$ by removing $S$, taking the closure $\overline{X-S}$ as in $\S 2$, and gluing in an $S^{1}$ invariant neighborhood of a surface $S^{+}$diffeomorphic to $S$. Again take the area of the fibers to be some sufficiently small $\varepsilon$. We denote the triple that arises from the thickening by $\varepsilon$ along $S$ by

$$
\left(\mathcal{T}_{S}^{+}(X), S^{+}, T^{+}\right)
$$

It is easy to see that this thickening is just given by summing with $W_{g, \varepsilon}$ :

$$
\left(\mathcal{T}_{S}^{+}(X), S^{+}, T^{+}\right)=\left(X \#_{S=\Gamma_{k}} W_{g, \varepsilon}, \Gamma_{-k}, F \# T\right) .
$$

Moreover, the symplectic structure $\omega^{+}$on this manifold has

$$
\int_{\mathcal{T}_{S}^{+}(X)}\left(\omega^{+}\right)^{2}>\int_{X} \omega^{2}, \quad \int_{S^{+}} \omega^{+}=\varepsilon \iota_{S}+\int_{S} \omega, \quad \int_{T^{+}} \omega^{+}=\varepsilon+\int_{T} \omega .
$$

We will use the following property of thickening and thinning in the proof of the associativity rule.

Lemma 4.2. If $\left(X_{i}, S_{i}, T_{i}\right), i=1,2$ are triples such that the sum

$$
\left(X_{1} \#_{T_{1}=S_{2}} X_{2}, S_{1} \# T_{2}\right)
$$

is defined, then for sufficiently small $\varepsilon$

$$
\left(\mathcal{T}_{T_{1}}^{-}\left(X_{1}\right) \#_{T_{1}^{-}=S_{2}^{+}} \mathcal{T}_{S_{2}}^{+}\left(X_{2}\right), S_{1}^{-} \# T_{2}^{+}\right)=\left(X_{1} \#_{T_{1}=S_{2}} X_{2}, S_{1} \# T_{2}\right) .
$$


Proof. This is clear, since it is just a matter of cutting points out of a neighborhood of $T_{1}$ in $X_{1}$ and moving them to a neighborhood of $S_{2}^{+}$in $\mathcal{T}_{S_{2}}^{+}\left(X_{2}\right)$. Explicitly, using the definitions of thickening and thinning, one can see that both sides are equal to

$$
\left(\mathcal{T}_{T_{1}}^{-}\left(X_{1}\right) \#_{T_{1}^{-}=\Gamma_{k}} W_{g, \varepsilon} \#_{\Gamma_{-k}=S_{2}} X_{2}, S_{1}^{-} \# F \# T_{2}\right)
$$

where $W_{g, \varepsilon}, g=g_{T_{1}}$ is a ruled surface with fiber $F$ and disjoint sections $\Gamma_{k}, \Gamma_{-k}$ where $k=-\iota_{T_{1}}$.

The refined version of Lemma 1.3 that we need is the following:

Lemma 4.3. Given any triple $(X, S, T)$ and some sufficiently small $\varepsilon$, let $k=-\iota_{S}$ and consider the manifold $W_{g, 2 \varepsilon}, g=g_{S}$, which has a section $\Gamma_{k}$ of area $A_{S}+(k+1) \varepsilon$ where $A_{S}$ is the symplectic area of $S$. Then

$$
\left(W_{g, 2 \varepsilon} \#_{\Gamma_{k}=\left(S^{-}\right)^{+}} \mathcal{T}_{T^{-}}^{+}\left(\mathcal{T}_{S}^{-}(X)\right), \Gamma_{-k+2} \#\left(T^{-}\right)^{+}\right)=\left(\mathcal{T}_{S+T}^{+}(X),(S+T)^{+}\right)
$$

where the thickening and thinning are by the amount $\varepsilon$. Similarly, if $(g, k)=\left(g_{T}, \iota_{T}+2\right)$ and the section $\Gamma_{-k+2}$ has area $A_{T}+(3-k) \varepsilon$ then

$$
\left(\mathcal{T}_{S^{-}}^{+}\left(\mathcal{T}_{T}^{-}(X)\right) \#_{\left(T^{-}\right)^{+}=\Gamma_{-k+2}} W_{g, 2 \varepsilon},\left(S^{-}\right)^{+} \# \Gamma_{k}\right)=\left(\mathcal{T}_{S+T}^{+}(X),(S+T)^{+}\right) .
$$

Proof. As before, the second statement follows from the first by replacing $k$ with $-k+2$ and interchanging $S, T$. We begin by proving the symplectic equivalence of the manifolds in the first statement, and then show how the submanifolds are also identified.

Observe that the definitions immediately imply

$$
\left(W_{g, \varepsilon} \#_{\Gamma_{k}=S^{-}} \mathcal{T}_{S}^{-}(X), \Gamma_{-k+2} \# T^{-}\right)=(X, S+T) .
$$

We need something more subtle. Define

$$
\begin{aligned}
& \left(X_{3}, S_{3}, T_{3}\right)=\left(\mathcal{T}_{S}^{-}(X), S^{-}, T^{-}\right) \\
& \left(X_{4}, S_{4}, T_{4}\right)=\left(W_{g^{\prime}, \varepsilon}, \Gamma_{k^{\prime}}, F^{\prime}\right)
\end{aligned}
$$

where $g^{\prime}=g_{T}, k^{\prime}=-\iota_{T}$ so that

$$
\left(\mathcal{T}_{T^{-}}^{+}\left(\mathcal{T}_{S}^{-}(X)\right),\left(S^{-}\right)^{+}\right)=\left(X_{3} \#_{T_{3}=S_{4}} X_{4}, S_{3} \# T_{4}\right) .
$$

The lemma then follows from the 4-fold sum rule (Proposition 1.1) once we express $W_{g, 2 \varepsilon}$ appropriately as a sum of two ruled surfaces $X_{1} \# X_{2}$ each with fibers $F^{i}$ of area $\varepsilon$. Namely, apply Lemma 4.1 using a Hamiltonian function that has fixed point sets $\Gamma_{-k+2}, \Gamma_{k-2}$ to define

$$
\begin{aligned}
& \left(X_{1}, S_{1}, T_{1}\right)=\left(W_{g, \varepsilon}^{1}, F^{1}, \Gamma_{k-2}^{1}\right) \\
& \left(X_{2}, S_{2}, T_{2}\right)=\left(W_{g, \varepsilon}^{2}, \Gamma_{-k+2}^{2}, \Gamma_{k}^{2}\right)
\end{aligned}
$$


where the areas of $T_{1}=\Gamma_{k-2}^{1}$ and $S_{2}=\Gamma_{-k+2}^{2}$ both equal $A_{S}+\varepsilon$. Then $S_{1} \# T_{2}$ has area $A_{S}+(k+1) \varepsilon$ and

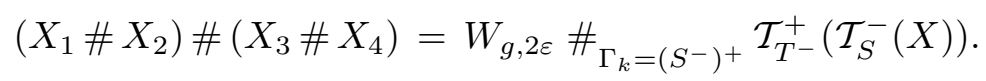

On the other hand

$$
X_{2} \# X_{3}=W_{g, \varepsilon}^{2} \#_{\Gamma_{k}=S^{-}} \mathcal{T}_{S}^{-}(X)=X
$$

and $X_{4} \# X_{1}$ is a ruled surface with fibers of area $\varepsilon$ over a Riemann surface of genus $g=g_{S}+g_{T}$. The surface $S_{2} \# T_{3}=\Gamma_{-k+2}^{2} \# T^{-}$has selfintersection $\iota_{S}+\iota_{T}+2$ and is in the class of $[S]+[T]$, so we can choose it as the representative $S+T$. It is not hard to check that summing with $X_{4} \# X_{1}$ simply thickens $X=X_{2} \# X_{3}$ along $S+T$ as desired, so

$$
\left(X_{4} \# X_{1}\right) \#\left(X_{2} \# X_{3}\right)=X_{S+T}^{+} .
$$

To see that the symplectomorphism of Proposition 1.1 identifies $\Gamma_{-k+2} \#\left(T^{-}\right)^{+}$and $(S+T)^{+}$, notice that $\left(T^{-}\right)^{+}$is in fact a section of $W_{4}$ disjoint from $S_{4}$, and $\Gamma_{-k+2}$ is a section of $W_{1}$ disjoint from $T_{1}$. Therefore, when we sum along the fibers of $X_{4}, X_{1}$ we get another ruled surface containing the connected sum of these sections, which is a section disjoint from $S_{4} \# T_{1}$ and therefore corresponds to $(S+T)^{+}$.

We are now ready to prove that the associativity rule holds up to symplectomorphism. Roughly, the strategy is to thin the manifolds $X_{i}$ and use the removed neighborhoods to create the necessary $X_{4}=W_{g, 2 \varepsilon}$ so as to apply the 4 -fold sum rule. To do this correctly, we need to do some thickening as well. Note that the order in which one thickens and thins does not matter, i.e.

$$
\mathcal{T}_{T^{-}}^{+}\left(\mathcal{T}_{S}^{-}(X)\right)=\mathcal{T}_{S^{+}}^{-}\left(\mathcal{T}_{T}^{+}(X)\right) .
$$

Proof of Proposition 1.2. We must show that

$$
\left(X_{1} \#_{T_{1}=S_{2}} X_{2}\right) \#_{S_{1} \# T_{2}=S_{3}+T_{3}} X_{3}=X_{1} \#_{S_{1}+T_{1}=S_{2} \# T_{3}}\left(X_{2} \#_{T_{2}=S_{3}} X_{3}\right)
$$

under the given hypotheses. According to the above strategy, consider the triples $\left(X_{i}^{\prime}, S_{i}^{\prime}, T_{i}^{\prime}\right), i=1, \ldots 3$ where we have thickened and thinned by a sufficiently small $\varepsilon$ to obtain

$$
X_{1}^{\prime}=\mathcal{T}_{T_{1}^{-}}^{+}\left(\mathcal{T}_{S_{1}}^{-}\left(X_{1}\right)\right), \quad X_{2}^{\prime}=\mathcal{T}_{T_{2}^{-}}^{-}\left(\mathcal{T}_{S_{2}}^{-}\left(X_{2}\right)\right), \quad X_{3}^{\prime}=\mathcal{T}_{S_{3}^{-}}^{+}\left(\mathcal{T}_{T_{3}}^{-}\left(X_{3}\right)\right)
$$

and surfaces $S_{i}^{\prime}, T_{i}^{\prime}$ which are the deformed $S_{i}, T_{i}$. Then choose

$$
\left(X_{4}^{\prime}, S_{4}^{\prime}, T_{4}^{\prime}\right)=\left(W_{g, 2 \varepsilon}, \Gamma_{-k+2}, \Gamma_{k}\right)
$$


where $k=-\iota_{S}$ and the areas of $S_{4}^{\prime}, T_{4}^{\prime}$ equal those of $T_{3}^{\prime}, S_{1}^{\prime}$. This choice is possible since in both cases the difference in the areas of the two submanifolds is $2(1-k) \varepsilon$. The triples are admissible for the 4 -fold sum, so the following calculations prove the proposition. We suppress the subscripts indicating the submanifolds along which the sums are being performed when there is no ambiguity. Invoking Lemmas 4.2 and 4.3 , we have

$$
\begin{aligned}
& \left(X_{1}^{\prime} \#_{T_{1}^{\prime}=S_{2}^{\prime}} X_{2}^{\prime}\right) \#_{S_{1}^{\prime} \# T_{2}^{\prime}=S_{3}^{\prime} \# T_{4}^{\prime}}\left(X_{3}^{\prime} \#_{T_{3}^{\prime}=S_{4}^{\prime}} X_{4}^{\prime}\right) \\
& =\left(\mathcal{T}_{T_{1}^{-}}^{+}\left(\mathcal{T}_{S_{1}}^{-}\left(X_{1}\right)\right) \# \mathcal{T}_{S_{2}^{-}}^{-}\left(\mathcal{T}_{T_{2}}^{-}\left(X_{2}\right)\right)\right) \#\left(\mathcal{T}_{S_{3}^{-}}^{+}\left(\mathcal{T}_{T_{3}}^{-}\left(X_{3}\right)\right) \# W_{g, 2 \varepsilon}\right) \\
& =\left(\mathcal{T}_{S_{1}}^{-}\left(X_{1}\right) \#_{T_{1}^{-}=S_{2}^{-}} \mathcal{T}_{T_{2}}^{-}\left(X_{2}\right)\right) \#_{S_{1}^{-} \# T_{2}^{-}=\left(S_{3}+T_{3}\right)^{+}} \mathcal{T}_{S_{3}+T_{3}}^{+}\left(X_{3}\right) \\
& =\left(X_{1} \#_{T_{1}=S_{2}} X_{2}\right) \#_{S_{1} \# T_{2}=S_{3}+T_{3}} X_{3}
\end{aligned}
$$

and

$$
\begin{aligned}
& \left(X_{4}^{\prime} \#_{T_{4}^{\prime}=S_{1}^{\prime}} X_{1}^{\prime}\right) \#_{S_{4}^{\prime} \# T_{1}^{\prime}=S_{2}^{\prime} \# T_{3}^{\prime}}\left(X_{2}^{\prime} \#_{T_{2}^{\prime}=S_{3}^{\prime}} X_{3}^{\prime}\right) \\
& =\left(W_{g, 2 \varepsilon} \# \mathcal{T}_{T_{1}^{-}}^{+}\left(\mathcal{T}_{S_{1}}^{-}\left(X_{1}\right)\right)\right) \#\left(\mathcal{T}_{T_{2}^{-}}^{-}\left(\mathcal{T}_{S_{2}}^{-}\left(X_{2}\right)\right) \# \mathcal{T}_{S_{3}^{-}}^{+}\left(\mathcal{T}_{T_{3}}^{-}\left(X_{3}\right)\right)\right) \\
& =\mathcal{T}_{S_{1}+T_{1}}^{+}\left(X_{1}\right) \#_{\left(S_{1}+T_{1}\right)^{+}=S_{2}^{-} \# T_{3}^{-}}\left(\mathcal{T}_{S_{2}}^{-}\left(X_{2}\right) \#_{T_{2}^{-}=S_{3}^{-}} \mathcal{T}_{T_{3}}^{-}\left(X_{3}\right)\right) \\
& =X_{1} \#_{S_{1}+T_{1}=S_{2} \# T_{3}}\left(X_{2} \#_{T_{2}=S_{3}} X_{3}\right)
\end{aligned}
$$

\section{Acknowledgements}

The first author wishes to thank Gompf for bringing these examples to her attention and the organizers of the Gökova conference for providing such a beautiful place in which to do research. The second author wishes to thank Yakov Eliashberg for his mathematical guidance and the Harvard mathematics department for providing a welcoming and stimulating workplace.

\section{References}

1. R. Gompf, A new construction of symplectic manifolds, Ann. of Math. 142 (1995), 527-595.

$2 . \quad$, private communication.

3. M. Gromov, Partial Differential Relations, Springer-Verlag, Berlin (1986).

4. F. Lalonde and D. McDuff, The classification of ruled symplectic 4-manifolds, Preprint (1995).

5. E. Lerman, Symplectic cuts, Math. Res. Lett. 2 (1995), 247-258.

6. J. McCarthy and J. Wolfson, Symplectic normal connect sum, Topology 33 (1994), 729-764. 
7. Y. Ruan, Symplectic topology on algebraic 3-folds, J. Diff. Geom. 39 (1994), $215-227$.

8. A. Stipsicz, private communication.

9. M. Symington, New constructions of symplectic four-manifolds, thesis, Stanford University (1996).

10. C. Taubes, The Seiberg-Witten and the Gromov invariants, Math. Res. Lett. 2 (1995), 221-238.

State University of New York at Stony Brook

E-mail address: dusa@math.sunysb.edu

StAnFord University, StAnFord, CA 94305

E-mail address: margaret@math.stanford.edu 